\title{
Association Between Habitual Green Tea Consumption and Comprehensive Frailty Risk Assessed by Kihon Checklist Indexes Among Japanese Elderly Population
}

Hinako Nanri ( $\nabla$ hnanri@nibiohn.go.jp )

National Institutes of Biomedical Innovation, Health and Nutrition

Tsukasa Yoshida

National Institutes of Biomedical Innovation, Health and Nutrition

Yuya Watanabe

Physical Fitness Research Institute, Meiji Yasuda Life Foundation of Health and Welfare

Hiroyuki Fujita

Kyoto University of Advanced Science

Misaka Kimura

Kyoto University of Advanced Science

\section{Yosuke Yamada}

National Institutes of Biomedical Innovation, Health and Nutrition

\section{Research Article}

Keywords: green tea, comprehensive frailty, Kihon check list, elderly, Japanese

Posted Date: August 13th, 2021

DOI: https://doi.org/10.21203/rs.3.rs-798460/v1

License: (c) (i) This work is licensed under a Creative Commons Attribution 4.0 International License.

Read Full License 


\section{Abstract}

Background: It is unclear whether habitual green tea consumption is related to comprehensive frailty.

Objective: We conducted this study to investigate this relationship among a Japanese elderly population.

Methods: This was a cross-sectional study of baseline data from 2012. The study included 5668 Japanese participants (2766 men and 2902 women, aged 65 years or older). The subjects completed a validated self-administered food frequency questionnaire that included green tea consumption. We evaluated comprehensive frailty by using a 25 -item Kihon Checklist (KCL), which comprised seven domains (instrumental activities of daily living [IADL], physical function, malnutrition, oral or eating function, socialization and housebound, cognitive function, and depression). Frailty was defined as a $\mathrm{KCL}$ score $\geq 7$.

Results: We found that higher consumption of green tea was associated with a lower risk of frailty in both sexes. Further age-stratified analysis showed that higher consumption of green tea among women was associated with a lower risk of frailty, regardless of age. In men, however, these associations were found only in the older age groups. An analysis of the association between green tea consumption and frailty subdomains showed that green tea consumption was associated with a lower risk of oral dysfunction and cognitive problem in both sexes. In addition, only in women, higher green tea consumption was inversely associated with a lower risk of IADL and mobility-related disability problems.

Conclusions: Green tea consumption is inversely associated with the prevalence of comprehensive frailty in Japanese men and women. Longitudinal studies are required to confirm this association.

\section{Introduction}

Tea, one of the most commonly consumed beverages worldwide, has beneficial effects on human health. ${ }^{1}$ Green tea in particular contains more polyphenol such as catechins, belonging to the group of flavonoids, than other types of tea (e.g. black and oolong tea) made from the same plant (Camellia sinensis) ${ }^{2}$. Catechins, especially (-)-epigallocatechin-3-gallate (EGCG), are known to act as antioxidants in vitro; they prevent oxidation or increase the cellular lipid antioxidant activity of vitamins $\mathrm{C}$ and $\mathrm{E}$ in humans. ${ }^{2}$ Numerous epidemiologic studies have reported an inverse association of green tea consumption with the risk of chronic inflammatory disease such as cancer. ${ }^{1}$ Additionally, in observational studies, habitual green tea consumption has been reported to have beneficial effects on age-related disorders. ${ }^{3,4}$

Frailty is an age-related physiological syndrome, and it increases health outcomes such as falls, hospitalization, and death; therefore, early prevention is critical. ${ }^{5}$ Although there are still some debates regarding the definition of frailty, the phenotype of physical frailty, as defined by Fried and colleagues, is characterized by unintentional weight loss, global muscle weakness, exhaustion or poor endurance, slowed performance, and low physical activity. ${ }^{6}$ More recently, frailty is considered to be multifaceted, 
with components such as physical frailty as well as psychological and sociological frailty. ${ }^{5}$ There is growing evidence about the association between frailty and chronic inflammation, ${ }^{7}$ pro-inflammatory cytokines may influence frailty either directly by promoting protein and degradation, or indirectly by affecting important metabolic pathways. ${ }^{8}$ In addition, accumulated epidemiological studies have reported the important role of dietary factors in preventing frailty. ${ }^{9}$

Several studies have reported inverse associations of frailty risk with foods and nutrients intake. ${ }^{10-12} \mathrm{~A}$ meta-analysis of four cohort studies reported that high adherence to a Mediterranean diet characterized by a high intake of antioxidants-rich fruits and vegetables is associated with significantly lower physical frailty risk. ${ }^{10}$ Previous studies have shown that antioxidants (i.e., vitamin $\mathrm{C}$, vitamin $\mathrm{E}$, and polyphenol) or dietary non-enzymatic antioxidant capacity were inversely associated with physical frailty risk. ${ }^{11,12}$ However, it is unclear whether the habitual consumption of green tea, which contains high antioxidants, is associated with comprehensive frailty. Furthermore, given that people with oxidative stress-related risk factors have a higher bioavailability of antioxidants than those without, ${ }^{13}$ the effect of green tea consumption on frailty may be greater in postmenopausal women, who are at a higher risk for chronic inflammation, than in men. The aim of the present study was to investigate the association between habitual green tea consumption and frailty by sex among Japanese elderly population. We also examined the relationship between green tea intake and the seven subdomains that constitute comprehensive frailty.

\section{Methods}

\section{Study subjects}

We analyzed baseline data from the population-based Kyoto-Kameoka Study, which aimed to examine the associations between food intake, nutritional status, physical activity, oral function and long-term care (LTC) insurance among the community-dwelling older people in Kyoto prefecture, Japan. ${ }^{14}$ The study participants and methods used in the Kyoto-Kameoka Study have been described in detail elsewhere. ${ }^{14}$ The Daily Life Area Needs Survey was conducted by mail among 18231 elderly people aged 65 years or older living in Kameoka City, Kyoto Prefecture (as of July 1, 2011), excluding those who required nursing care levels is 3 or more, and 13159 of them responded (response rate: $73.2 \%)$. Second, after excluding 69 people who died and those who required support or care, an additional survey of 12054 people was conducted in February 2012, and the number of respondents was 8,319 (response rate: $69.4 \%)$.

From the 8319 participants, we further excluded 2681 who met any of the following conditions: missing data on the consumption of green tea $(n=375)$ and Kihon Checklist score $(n=2276)$. Finally, 5668 participants were eligible for this study (2766 men and 2902 women). We described the study concept in the mail survey. Participants gave informed consent by returning the questionnaire with the participant's name. The entire study protocol was reviewed and approved the Ethics Committees of Kyoto Prefectural 
University of Medicine (RBMR-E-363), Kyoto University of Advanced Science (No. 20-1), and the National Institutes of Biomedical Innovation, Health and Nutrition (NIBIOHN-76-2) and that it conforms to the provisions of the Declaration of Helsinki.

The entire study protocol was reviewed and approved the Ethics Committees of Kyoto Prefectural University of Medicine (RBMR-E-363), Kyoto University of Advanced Science (No. 20-1), and the National Institutes of Biomedical Innovation, Health and Nutrition (NIBIOHN-76-2) and that it conforms to the provisions of the Declaration of Helsinki. Participants gave informed consent by returning the questionnaire with the participant's name.

\section{Measurements}

\section{Dietary assessment}

Dietary intake was assessed using a commonly used previously validated Japanese food frequency questionnaire (FFQ). ${ }^{15,16}$ In this FFQ, we asked participants to report their intake frequency to assess the average intakes of 46 foods and beverages (green tea and coffee) items over the past year. The frequencies of green tea consumption were categorized as follows: almost none, 1 to 3 times/month, 1 to 2 times/week, 3 to 4 times/week, 5 to 6 times/week, 1 time/day, 2 times/day, and $\geq 3$ times/day. The total energy intake was calculated using a program developed by Tokudome et al, ${ }^{15,16}$ based on the standard tables of food consumption In Japan (fifth revised edition). ${ }^{17}$

\section{Definition of frailty}

In Japan, the Kihon Checklist (KCL) was developed by the Japanese Ministry of Health, Labor and Welfare to screen for future risks of LTC certification. ${ }^{18,19}$ Recently, the KCL was validated for use in screening community-dwelling older residents for frailty, ${ }^{18}$ it was translated to other languages and used in various countries. ${ }^{20}$ The strengths of the $\mathrm{KCL}$ include its use in the assessment of physical, sociological, and psychological domains as a comprehensive questionnaire: 1) questions 1-5 assessed "instrumental activities of daily living (IADL)", 2) questions 6-10 "physical function/strength", 3) questions 11-12 "malnutrition", 4) questions 13-15 "oral function/eating", 5) questions 16-17 "socialization/housebound", 6) questions 18-20 cognitive/memory, and 7) questions 21-25 "depression/mood". ${ }^{19}$ When the dichotomous response (yes / no) fell under a risk of frailty, the score is pointed +1 . A high $\mathrm{KCL}$ score (range $0-25$ ) indicates worse functioning and severe frailty. The total score of original $\mathrm{KCL} \geq 7$ points was indicated a higher risk of long-term care. ${ }^{21}$ The cut-off point at which problems were defined for the following seven domains are as follows: 1) IADL score $\geq 3$ points, 2) mobility disability $\geq 3$ points, 3 ) nutrition 2 points, 4 ) oral or eating function $\geq 2$ points, 5) socialization 
and housebound problems were indicated in people who answered 'no' to Q16 (Do you go out at least once a week?), 6) cognitive function $\geq 1$ points, and 7) depression $\geq 2$ points.

\section{Other variables}

A self-administered questionnaire was used to assess height, weight, alcohol consumption status, and smoking status. Alcohol consumption status was classified into the following categories: everyday, sometimes, seldom, and never. Smoking status was classified into the following: everyday, sometimes, formerly, and never. BMI was calculated as the self-reported body weight (in $\mathrm{kg}$ ) divided by the square of the self-reported height (in $\mathrm{m}^{2}$ ).

\section{Statistical analysis}

All statistical analyses were performed separately for men and women with the SAS statistical software package (Ver. 9.4 for Windows; SAS Institute, Cary, NC, USA). We categorized their green tea consumption based on their beverages consumption distribution as follows: almost none, $<1$ cup/day, 1-2 cups/day, $\geq 3$ cups/day for green tea. The participants' characteristics according to categories of green tea consumption were compared by linear regression analysis (for continuous variables) or MantelHaenszel test (for categorical variables), as appropriate. Adjusted odds ratio (ORs) and 95\% confidence intervals (Cls) for frailty risk according to the consumption of green tea was estimated using logistic regression analysis. Adjustments were made for age (years, continuous), BMI ( $\mathrm{kg} / \mathrm{m}^{2}$, continuous), total energy intake (kcal/day, continuous), alcohol consumption status (everyday, sometimes, seldom, or never), smoking status (everyday, sometimes, formerly, or never). We found that subjects with higher consumption of green tea were more likely to consume coffee. We further adjusted for coffee consumption (almost none, $<1$ cup/day, 1 cup/day, or $\geq 2$ cups/day) in each model as covariate. A $P$ value $<0.05$ was considered statistically significant.

\section{Results}

Table 1 shows the characteristics of the participants stratified by sex. The proportion of current drinkers or current smokers and the mean total energy intake were higher among men than among women, whereas the average age, proportion of those who were underweight, and the intake frequency of fruits and vegetables were higher among women than among men. The mean total $\mathrm{KCL}$ scores was 5.0 for men and 5.1 for women, and the prevalence of KCL frailty risk (total KCL score $\geq 7$ ) was $29.3 \%$ in men and $30.6 \%$ in women, which were non statistically significant difference between both sexes. The proportion of those with IADL and cognitive and memory problems was higher among men than among women, whereas the proportion of those with physical function/strength problem, malnutrition problem, socialization and housebound problem were higher among women than among men. 
Table 1

Characteristics in the study subjects in men and women.

\begin{tabular}{|c|c|c|c|}
\hline & Men & Women & \multirow[t]{2}{*}{ Pvalue * } \\
\hline & $(n=2766)$ & $(n=2902)$ & \\
\hline Age (years) & $73.1(5.7)^{\dagger}$ & $73.5(6.0)$ & 0.01 \\
\hline$<75$ years & $1798(65.0)$ & $1828(63.0)$ & \multirow[t]{2}{*}{0.11} \\
\hline$\geq 75$ years & $968(35.0)$ & $1074(37.0)$ & \\
\hline Body mass index $\left(\mathrm{kg} / \mathrm{m}^{2}\right)$ & $23.1(2.7)$ & $22.5(3.3)$ & $<0.001$ \\
\hline Underweight, $<18.5$ & $109(4.0)$ & $266(9.2)$ & \multirow[t]{3}{*}{$<0.001$} \\
\hline Normal weight, 18.5-24.9 & $2040(74.2)$ & $2053(71.3)$ & \\
\hline Overweight, $\geq 25.0$ & $602(21.9)$ & $562(19.5)$ & \\
\hline \multicolumn{4}{|l|}{ Current drinker, n (\%) } \\
\hline Everyday & $1407(51.1)$ & $178(6.2)$ & \multirow[t]{4}{*}{$<0.001$} \\
\hline Sometimes & $456(16.6)$ & $431(14.9)$ & \\
\hline Almost never drink & $509(18.5)$ & $819(28.3)$ & \\
\hline Never & $380(13.8)$ & $1462(50.6)$ & \\
\hline \multicolumn{4}{|l|}{ Current smoker, n (\%) } \\
\hline Everyday & $459(16.6)$ & $81(2.8)$ & \multirow[t]{4}{*}{$<0.001$} \\
\hline Sometimes & $69(2.5)$ & $18(0.6)$ & \\
\hline Almost never smoke & $1655(60.0)$ & $199(6.9)$ & \\
\hline Never & $577(21.0)$ & $2574(89.6)$ & \\
\hline Total energy intake (kcal/day) & $1915(525)$ & 1577 (333) & $<0.001$ \\
\hline
\end{tabular}

$\mathrm{KCL}$, Kihon checklist; IADL, Instrumental activities of daily living.

${ }^{*} P$ values for sex difference are based on $t$ test for continuous variables and chi-square tests for categorical variables.

+ Values are mean (standard deviation) for continuous variables and number (percentage) for categorical variables.

variables.

‡ People who answer 'no' at Q16 has socialization/housebound problems. 


\begin{tabular}{|c|c|c|c|}
\hline & Men & Women & $P$ value * \\
\hline & $(n=2766)$ & $(n=2902)$ & \\
\hline Fruit intake (times/day) & $0.79(0.79)$ & $1.11(0.92)$ & $<0.001$ \\
\hline Vegetable intake (times/day) & $3.28(2.28)$ & $4.11(2.65)$ & $<0.001$ \\
\hline Coffee consumption $\geq 1$ cup/day, n (\%) & $1008(35.0)$ & $1876(65.1)$ & 0.025 \\
\hline Total KCL score, (range: $0-25$ ) & $5.0(4.1)$ & $5.1(4.3)$ & 0.09 \\
\hline Comprehensive frailty based on KCL score & $810(29.3)$ & $889(30.6)$ & 0.27 \\
\hline \multicolumn{4}{|l|}{ KCL subdomains } \\
\hline People with IADL problem & $396(14.3)$ & $270(9.3)$ & $<0.001$ \\
\hline People with physical function/strength problem & $398(14.4)$ & $715(24.6)$ & $<0.001$ \\
\hline People with have malnutrition problem & $35(1.3)$ & $62(2.1)$ & 0.01 \\
\hline People with oral function/eating problem & $603(21.8)$ & $589(20.3)$ & 0.16 \\
\hline People with socialization/housebound problem ${ }^{\ddagger}$ & $133(4.8)$ & $222(7.7)$ & $<0.001$ \\
\hline People with cognitive/memory problem & $254(9.2)$ & $188(6.5)$ & $<0.001$ \\
\hline People with depression/mood problem & $730(26.3)$ & $823(28.4)$ & 0.10 \\
\hline \multicolumn{4}{|c|}{$\mathrm{KCL}$, Kihon checklist; IADL, Instrumental activities of daily living. } \\
\hline \multicolumn{4}{|c|}{$\begin{array}{l}{ }^{*} P \text { values for sex difference are based on } t \text { test for continuous variables and chi-square tests for } \\
\text { categorical variables. }\end{array}$} \\
\hline \multicolumn{4}{|c|}{$\begin{array}{l}\text { † Values are mean (standard deviation) for continuous variables and number (percentage) for } \\
\text { categorical variables. }\end{array}$} \\
\hline \multicolumn{4}{|l|}{ variables. } \\
\hline
\end{tabular}

Table 2 shows the basic characteristics of the study participants according to their consumption of green tea. Men who consumed more green tea tended to be older, have a higher total energy intake and more frequent fruit and vegetable intake, and higher coffee consumption, and they tended to have a lower proportion of current smokers. Women who consumed more green tea tended to be younger, and other factors were similar associations in men. 
Table 2

Characteristics of the study subjects according to categories of green tea consumption.

\begin{tabular}{|c|c|c|c|c|c|}
\hline & \multicolumn{4}{|c|}{ Green tea consumption } & \multirow{2}{*}{$\begin{array}{l}P \text { for } \\
\text { trend * }\end{array}$} \\
\hline & None & $\begin{array}{l}<1 \\
\text { cup/day }\end{array}$ & $\begin{array}{l}1-2 \\
\text { cups/day }\end{array}$ & $\begin{array}{l}\geq 3 \\
\text { cups/day }\end{array}$ & \\
\hline Men, n (\%) & $\begin{array}{l}445 \\
(16.1)^{2}\end{array}$ & $\begin{array}{l}850 \\
(30.7)\end{array}$ & $699(25.3)$ & 772 (27.9) & \\
\hline Age (years) & $\begin{array}{l}72.4 \\
(5.4)\end{array}$ & $\begin{array}{l}72.3 \\
(5.3)\end{array}$ & $73.5(5.8)$ & $74.0(6.0)$ & $<0.001$ \\
\hline Body mass index $\left(\mathrm{kg} / \mathrm{m}^{2}\right)$ & $\begin{array}{l}23.1 \\
(2.7)\end{array}$ & $\begin{array}{l}23.2 \\
(2.8)\end{array}$ & $23.3(3.0)$ & $23.0(2.7)$ & 0.34 \\
\hline Current drinker, $\mathrm{n}(\%)$ & $\begin{array}{l}307 \\
(69.3)\end{array}$ & $\begin{array}{l}579 \\
(68.5)\end{array}$ & $475(68.2)$ & $502(65.4)$ & 0.054 \\
\hline Current smoker, n (\%) & $\begin{array}{l}110 \\
(24.7)\end{array}$ & $\begin{array}{l}176 \\
(20.7)\end{array}$ & $121(17.4)$ & $121(15.7)$ & $<0.001$ \\
\hline Total energy intake (kcal/day) & $\begin{array}{l}1833 \\
(573)\end{array}$ & $\begin{array}{l}1885 \\
(538)\end{array}$ & 1899 (498) & $\begin{array}{l}2010 \\
(492)\end{array}$ & $<0.001$ \\
\hline Fruit intake (times/day) & $\begin{array}{l}0.63 \\
(0.75)\end{array}$ & $\begin{array}{l}0.68 \\
(0.65)\end{array}$ & $0.85(0.75)$ & $\begin{array}{l}0.95 \\
(0.95)\end{array}$ & $<0.001$ \\
\hline Vegetable intake (times/day) & $\begin{array}{l}2.76 \\
(1.99)\end{array}$ & $\begin{array}{l}2.84 \\
(1.73)\end{array}$ & $3.39(2.05)$ & $\begin{array}{l}3.98 \\
(2.89)\end{array}$ & $<0.001$ \\
\hline $\begin{array}{l}\text { Coffee consumption } \geq 1 \\
\text { cup/day, } n(\%)\end{array}$ & $\begin{array}{l}259 \\
(58.3)\end{array}$ & $\begin{array}{l}448 \\
(52.8)\end{array}$ & $520(74.7)$ & $486(63.4)$ & $<0.001$ \\
\hline Women, n (\%) & $282(9.7)$ & $\begin{array}{l}625 \\
(21.5)\end{array}$ & $763(26.3)$ & $\begin{array}{l}1232 \\
(42.6)\end{array}$ & \\
\hline Age (years) & $\begin{array}{l}74.0 \\
(6.6)\end{array}$ & $\begin{array}{l}73.1 \\
(5.8)\end{array}$ & $73.0(5.8)$ & $73.8(6.1)$ & 0.006 \\
\hline Body mass index $\left(\mathrm{kg} / \mathrm{m}^{2}\right)$ & $\begin{array}{l}22.6 \\
(3.7)\end{array}$ & $\begin{array}{l}22.6 \\
(3.3)\end{array}$ & $22.5(3.2)$ & $22.3(3.3)$ & 0.34 \\
\hline Current drinker, $\mathrm{n}(\%)$ & $52(18.5)$ & $\begin{array}{l}133 \\
(21.5)\end{array}$ & $182(23.9)$ & $242(19.7)$ & 0.51 \\
\hline Current smoker, n (\%) & $13(4.7)$ & $32(5.2)$ & $23(3.0)$ & $31(2.5)$ & $<0.001$ \\
\hline
\end{tabular}

\footnotetext{
${ }^{*} P$ values for linear trends across quartiles (assigned ordinal numbers $0-3$ ) of green tea consumption are based on linear regression analysis for continuous variables and the Mantel test for categorical variables.
}

† Values are mean (standard deviation) for continuous variables and number (percentage) for categorical variables. 


\begin{tabular}{|c|c|c|c|c|c|}
\hline & \multicolumn{4}{|c|}{ Green tea consumption } & \multirow{2}{*}{$\begin{array}{l}\text { Pfor } \\
\text { trend * }\end{array}$} \\
\hline & None & $\begin{array}{l}<1 \\
\text { cup/day }\end{array}$ & $\begin{array}{l}1-2 \\
\text { cups/day }\end{array}$ & $\begin{array}{l}\geq 3 \\
\text { cups/day }\end{array}$ & \\
\hline Total energy intake (kcal/day) & $\begin{array}{l}1498 \\
(387)\end{array}$ & $\begin{array}{l}1518 \\
(331)\end{array}$ & $1552(310)$ & $\begin{array}{l}1638 \\
(320)\end{array}$ & $<0.001$ \\
\hline Fruit intake (times/day) & $\begin{array}{l}0.89 \\
(0.93)\end{array}$ & $\begin{array}{l}0.90 \\
(0.73)\end{array}$ & $1.14(0.84)$ & $\begin{array}{l}1.24 \\
(1.02)\end{array}$ & $<0.001$ \\
\hline Vegetable intake (times/day) & $\begin{array}{l}3.48 \\
(2.97)\end{array}$ & $\begin{array}{l}3.58 \\
(2.09)\end{array}$ & $4.07(2.21)$ & $\begin{array}{l}4.54 \\
(2.97)\end{array}$ & $<0.001$ \\
\hline $\begin{array}{l}\text { Coffee consumption } \geq 1 \\
\text { cup/day, } n(\%)\end{array}$ & $\begin{array}{l}146 \\
(52.1)\end{array}$ & $\begin{array}{l}345 \\
(55.7)\end{array}$ & $574(75.4)$ & $811(66.3)$ & $<0.001$ \\
\hline \multicolumn{6}{|c|}{$\begin{array}{l}{ }^{*} P \text { values for linear trends across quartiles (assigned ordinal numbers } 0-3 \text { ) of green tea consumption } \\
\text { are based on linear regression analysis for continuous variables and the Mantel test for categorical } \\
\text { variables. }\end{array}$} \\
\hline
\end{tabular}

Tables 3 shows multivariate-adjusted ORs and 95\% Cls for frailty risk according to green tea consumption. Green tea consumption was associated with a statistically significantly lower risk for frailty in both sexes ( $P$ for trend $=0.02$ in men and $<0.01$ in women). Men who consumed green tea $\geq 3$ cups/day had a lower risk of frailty than those who had almost none (ORs $=0.71,95 \% \mathrm{Cl}=0.54-0.94)$. Among women, there was a significantly reduced risk of frailty in all green tea consumption groups compared to those who had almost none (for $<1 \mathrm{cup} /$ day, $\mathrm{OR}=0.67,95 \% \mathrm{Cl}=0.49-0.92$; for $1-2$ cups $/$ day, $\mathrm{OR}=0.51,95 \% \mathrm{Cl}=0.37-0.70$; and for $\geq 3$ cups/day, $\mathrm{OR}=0.60,95 \% \mathrm{Cl}=0.44-0.81)$. In an agestratified analysis, among men, there was a statistically significant interaction between age and green tea consumption for frailty risk ( $P$ for interaction $=0.01$ ). Inverse association with frailty risk was observed for green tea consumption among those in the $\geq 75$ years age group ( $P$ for trend $<0.01$ ); green tea consumption $\geq 3$ cups/day was associated with a lower risk of frailty, compared with the consumption of almost none $(\mathrm{ORs}=0.46,95 \% \mathrm{Cl}=0.30-0.72)$. Meanwhile, there was no such association for those in the $<75$ years age group ( $P$ for trend $=0.57$ ). In women aged $<75$ years group, the risk of frailty was significantly reduced in all consumption groups compared with those who had almost none ( $<1 \mathrm{cup} /$ day, $\mathrm{OR}=0.66,95 \% \mathrm{Cl}=0.44-0.99 ; 1-2$ cups $/$ day, $\mathrm{OR}=0.41,95 \% \mathrm{Cl}=0.27-0.62 ;$ and $\geq 3$ cups $/$ day, $\mathrm{OR}=$ $0.51,95 \% \mathrm{Cl}=0.35-0.75)$. In women aged 75 years and older, the risk of frailty was significantly reduced in the $1-2$ cups $/$ day $(O R=0.60,95 \% \mathrm{Cl}=0.37-0.995)$ and $\geq 3$ cups $/$ day $(O R=0.61,95 \% \mathrm{Cl}=0.38-0.97)$ consumption categories. 
Table 3

Multivariated-adjusted odds ratios (OR) and $95 \%$ confidence intervals $(\mathrm{Cl})$ of comprehensive frailty according to categories of green tea consumption in men and women.

\begin{tabular}{|c|c|c|c|c|c|c|c|}
\hline & & \multicolumn{4}{|c|}{ Green tea consumption } & \multirow{2}{*}{$\begin{array}{l}P \text { for } \\
\text { trend }\end{array}$} & \multirow{2}{*}{$\begin{array}{l}P \text { for } \\
\text { Interaction }^{\dagger}\end{array}$} \\
\hline & & None & $\begin{array}{l}<1 \\
\text { cup/day }\end{array}$ & $\begin{array}{l}1-2 \\
\text { cups/day }\end{array}$ & $\begin{array}{l}\geq 3 \\
\text { cups/day }\end{array}$ & & \\
\hline \multirow[t]{9}{*}{ Men } & All, $n$ & 455 & 850 & 699 & 722 & & \\
\hline & $\begin{array}{l}\text { No. of } \\
\text { frailty (\%) }\end{array}$ & $\begin{array}{l}136 \\
(30.6)\end{array}$ & $255(30.0)$ & $222(31.8)$ & $197(25.5)$ & & \\
\hline & $\begin{array}{l}\mathrm{OR}(95 \% \\
\mathrm{Cl})\end{array}$ & $\begin{array}{l}1.00 \\
\text { (ref) }\end{array}$ & $\begin{array}{l}0.96 \\
(0.74- \\
1.25)^{\ddagger}\end{array}$ & $\begin{array}{l}1.07(0.82- \\
1.41)\end{array}$ & $\begin{array}{l}0.71 \\
(0.54- \\
0.94)\end{array}$ & 0.02 & \\
\hline & $\begin{array}{l}<75 \\
\text { years, } \mathrm{n}\end{array}$ & 317 & 599 & 436 & 444 & & \multirow[t]{6}{*}{0.01} \\
\hline & $\begin{array}{l}\text { No. of } \\
\text { frailty (\%) }\end{array}$ & $\begin{array}{l}71 \\
(22.4)\end{array}$ & $138(23.0)$ & $110(27.0)$ & $88(21.6)$ & & \\
\hline & $\begin{array}{l}\mathrm{OR}(95 \% \\
\mathrm{Cl})\end{array}$ & $\begin{array}{l}1.00 \\
\text { (ref) }\end{array}$ & $\begin{array}{l}1.06 \\
(0.76- \\
1.48)\end{array}$ & $\begin{array}{l}1.24(0.87- \\
1.76)\end{array}$ & $\begin{array}{l}0.87 \\
(0.60- \\
1.25)\end{array}$ & 0.57 & \\
\hline & $\begin{array}{l}\geq 75 \\
\text { years, } n\end{array}$ & 127 & 250 & 110 & 107 & & \\
\hline & $\begin{array}{l}\text { No. of } \\
\text { frailty (\%) }\end{array}$ & $\begin{array}{l}63 \\
(49.2)\end{array}$ & $112(44.6)$ & $111(42.5)$ & $107(32.6)$ & & \\
\hline & $\begin{array}{l}\mathrm{OR}(95 \% \\
\mathrm{Cl})\end{array}$ & $\begin{array}{l}1.00 \\
\text { (ref) }\end{array}$ & $\begin{array}{l}0.78 \\
(0.49- \\
1.24)\end{array}$ & $\begin{array}{l}0.77(0.49- \\
1.22)\end{array}$ & $\begin{array}{l}0.46 \\
(0.30- \\
0.72)\end{array}$ & $<0.01$ & \\
\hline \multirow[t]{2}{*}{ Women } & All, $n$ & 282 & 625 & 763 & 1232 & & \\
\hline & $\begin{array}{l}\text { No. of } \\
\text { frailty (\%) }\end{array}$ & $\begin{array}{l}127 \\
(45.0)\end{array}$ & $205(32.8)$ & $193(25.3)$ & $364(29.6)$ & & \\
\hline
\end{tabular}

KCL, Kihon checklist. IADL, instrumental activities of daily living.

*Based on multiple logistic regression analysis assigning ordinal numbers $0-3$ to quartile categories of protein intake.

${ }^{+}$Multiplicative interactions between green tea consumption and age groups.

‡ Adjusted for age (years), body mass index $\left(\mathrm{kg} / \mathrm{m}^{2}\right)$, total energy intake ( $\mathrm{kcal} /$ day), alcohol status (everyday, sometimes, seldom, or never), smoking status (everyday, sometimes, former, or never), vegetables intake, fruits intake, and coffee consumption (almost none, $<1$ cup/day, $1-2$ cups/day, $\geq$ 3 cups/day). 


\begin{tabular}{|c|c|c|c|c|c|c|}
\hline & \multicolumn{4}{|c|}{ Green tea consumption } & \multirow{2}{*}{$\begin{array}{l}P \text { for } \\
\text { trend }^{*}\end{array}$} & \multirow{2}{*}{$\begin{array}{l}P \text { for } \\
\text { Interaction }^{\dagger}\end{array}$} \\
\hline & None & $\begin{array}{l}<1 \\
\text { cup/day }\end{array}$ & $\begin{array}{l}1-2 \\
\text { cups/day }\end{array}$ & $\begin{array}{l}\geq 3 \\
\text { cups/day }\end{array}$ & & \\
\hline $\begin{array}{l}\text { OR }(95 \% \\
\mathrm{Cl})\end{array}$ & $\begin{array}{l}1.00 \\
\text { (ref) }\end{array}$ & $\begin{array}{l}0.67 \\
(0.49- \\
0.92)\end{array}$ & $\begin{array}{l}0.51(0.37- \\
0.70)\end{array}$ & $\begin{array}{l}0.60 \\
(0.44- \\
0.81)\end{array}$ & $<0.01$ & \\
\hline $\begin{array}{l}<75 \\
\text { years, } \mathrm{n}\end{array}$ & 317 & 598 & 436 & 444 & & 0.15 \\
\hline $\begin{array}{l}\text { No. of } \\
\text { frailty (\%) }\end{array}$ & $\begin{array}{l}57 \\
(33.1)\end{array}$ & $98(24.0)$ & $76(15.2)$ & $138(18.5)$ & & \\
\hline $\begin{array}{l}\mathrm{OR}(95 \% \\
\mathrm{Cl})\end{array}$ & $\begin{array}{l}1.00 \\
\text { (ref) }\end{array}$ & $\begin{array}{l}0.66 \\
(0.44- \\
0.99)\end{array}$ & $\begin{array}{l}0.41(0.27- \\
0.62)\end{array}$ & $\begin{array}{l}0.51 \\
(0.35- \\
0.75)\end{array}$ & $<0.01$ & \\
\hline $\begin{array}{l}\geq 75 \\
\text { years, } n\end{array}$ & 110 & 217 & 262 & 485 & & \\
\hline $\begin{array}{l}\text { No. of } \\
\text { frailty (\%) }\end{array}$ & $\begin{array}{l}68 \\
(61.8)\end{array}$ & $104(47.9)$ & $114(44.5)$ & $220(45.4)$ & & \\
\hline $\begin{array}{l}\mathrm{OR}(95 \% \\
\mathrm{Cl})\end{array}$ & $\begin{array}{l}1.00 \\
\text { (ref) }\end{array}$ & $\begin{array}{l}0.68 \\
(0.41- \\
1.13)\end{array}$ & $\begin{array}{l}0.60(0.37- \\
0.995)\end{array}$ & $\begin{array}{l}0.61 \\
(0.38- \\
0.97)\end{array}$ & 0.076 & \\
\hline \multicolumn{7}{|c|}{$K C L$, Kihon checklist. IADL, instrumental activities of daily living. } \\
\hline \multicolumn{7}{|c|}{$\begin{array}{l}\text { *Based on multiple logistic regression analysis assigning ordinal numbers } 0-3 \text { to quartile categories } \\
\text { of protein intake. }\end{array}$} \\
\hline \multicolumn{7}{|c|}{${ }^{\dagger}$ Multiplicative interactions between green tea consumption and age groups. } \\
\hline $\begin{array}{l}\text { ₹ Adjusted for age (yea } \\
\text { (everyday, sometimes, } \\
\text { vegetables intake, frui } \\
3 \text { cups/day). }\end{array}$ & $\begin{array}{l}\text {, body } \\
\text { Idom, } \\
\text { ntake, }\end{array}$ & $\begin{array}{l}\text { ass index } \\
\text { lever), smo } \\
\text { d coffee cc }\end{array}$ & $\begin{array}{l}n^{2} \text { ), total en } \\
\text { status (ev } \\
\text { mption (aln }\end{array}$ & $\begin{array}{l}\text { intake }(k \\
\text { y, someti } \\
\text { none, }<1\end{array}$ & $\begin{array}{l}\text { day), alc } \\
\text { forme } \\
\text { /day, } 1\end{array}$ & $\begin{array}{l}\text { I status } \\
\text { lever), } \\
\text { ips/day, } \geq\end{array}$ \\
\hline
\end{tabular}

The results for the seven subdomain risks (Fig. 1) showed a significant inverse association between green tea consumption and oral dysfunction ( $P$ for trend $=0.02$ in men and $P<0.01$ in women) and cognitive problem ( $P$ for trend $=0.02$ in men and $P<0.01$ in women) in both sexes. In women only, there were inverse associations between green tea consumption and IADL $(P$ for trend $<0.01)$ and mobilityrelated disability problems $(P$ for trend $=0.01)$.

\section{Discussion}


In a large population-based cohort study of older adults, we found that higher consumption of green tea was associated with a lower risk of frailty in both sexes. Further age-stratified analysis showed that, women who consumed more green tea had a lower risk of frailty, regardless of age. In men, however, these associations were found only in the older age groups. An analysis of the association between green tea consumption and frailty subdomains showed that, among both men and women, green tea consumption was associated with a lower risk of oral dysfunction and cognitive problem. In addition, only in women, higher green tea consumption was inversely associated with a lower risk of IADL and mobility-related disability problems. To our knowledge, this is the first observational study of the association between green tea consumption and comprehensive frailty risk.

Although there are currently no studies with which we can directly compare our results, our findings agreed with those from previous reports on associations between antioxidant nutrient intake and frailty risk. ${ }^{11,12,22}$ The Invecchiare in Chianti study reported that participants in the lowest quintile of vitamin $\mathrm{C}$ intake (OR, 2.12; 95\% Cl, 1.34-3.36; $P$ for trend $=0.001)$ and vitamin $\mathrm{E}$ intake (OR, 1.96; 95\% Cl, 1.25-3.07; $P$ for trend $=0.004$ ) had an increased risk of frailty compared with those in the highest quintile. ${ }^{22}$ Rabassa et al, in a longitudinal study, found that high habitual dietary resveratrol exposure was associated with a lower risk of frailty. ${ }^{11}$ Moreover, Kobayashi et al reported that higher dietary nonenzymatic antioxidant capacity was inversely associated with frailty risk. ${ }^{12}$

In the present study, in women, a higher consumption of green tea was associated with a lower risk of frailty, regardless of age. It has been reported that a decrease in sex hormones in postmenopausal women increases the risk of chronic inflammation with increased levels of $\mathrm{C}$-reactive protein and interleukin-6. ${ }^{13}$ Chronic inflammation has been shown to promote proteolysis and muscle catabolism, which in turn promotes increased risk of frailty. ${ }^{23}$ In fact, the previous study has shown that women become frailty at a younger age than men. ${ }^{6}$ We suggested that habitual intake of green tea, with its high antioxidant effect, may prevent frailty in women from an early stage.

Although the mechanism of the beneficial effect of green tea consumption on frailty risk remains unclear; there are possible biological explanations. Green tea polyphenols, mainly catechins, have been shown to protect humans against oxidative stress and inflammation, which can cause muscle atrophy and muscle fiber loss. ${ }^{24}$ EGCG have also been shown to increase antioxidant activity in animal models, and thus enhancing the overall chemo-preventative effect of antioxidants in those cells and tissues ${ }^{25}$. Additionally, green tea consumption can have beneficial effect on oral health; an in vivo study suggested that green tea catechins inhibit the growth of $P$. gingivalis. ${ }^{26}$ In addition, green tea consumption was associated with a higher general oral health assessment index. ${ }^{3}$ Maintaining good oral health can help prevent malnutrition, weight loss, and decline in social activities. ${ }^{27}$ Moreover, among Japanese population, green tea is considered an important source of certain nutrients such as antioxidant vitamins or polyphenols but also a main constituent of beverage intake. ${ }^{28}$ An observational study suggests that good hydration status prevents the risk of cognitive function decline. ${ }^{29}$ Moreover, higher consumption of water was 
positively associated with a decline in oral-related quality of life ${ }^{30}$. These results support the findings of our data that there is an association between green tea consumption and frailty subdomains.

Our study had some limitations. First, because the present study was cross-sectional, we were unable to conclude that the observed associations were causal. Second, the assessments of green tea consumption and comprehensive frailty were based on a self-administered questionnaire, which raises the problem of potential misclassification. Third, despite making adjustment for main potential confounders, the possibility of residual confounding cannot be ruled out. Fourth, the number of nutrition problems, one of the components of comprehensive frailty, was relatively small. That could lead to low statistical power in detecting significant associations. Finally, the present study was conducted only among a Japanese population. The ingredients of Japanese green tea could differ from those green tea types in other countries. Therefore, the findings of this study may not apply to other populations.

In conclusion, the present study confirmed that green tea consumption is inversely associated with the prevalence of comprehensive frailty. Future longitudinal studies are required to confirm habitual green tea consumption can predict future of development of frailty.

\section{Declarations}

\section{Acknowledgements}

This study was conducted with JSPS KAKENHI and was supported by a research grant provided to Misaka Kimura (24240091), Yosuke Yamada (15H05363) and Hinako Nanri (20K10547). We would like to thank all members of the Kyoto-Kameoka study Group for their valuable contributions. We acknowledge several administrative staffs of Kameoka city and Kyoto prefecture. We thank Shinkan Tokudome, who was a former director of the National Institute of Nutrition and Health.

\section{Disclosure statement}

The authors declare that they have no competing interests.

\section{References}

1. Abe, S. K. \& Inoue, M. Green tea and cancer and cardiometabolic diseases: a review of the current epidemiological evidence.Eur J Clin Nutr2020.

2. Carlson, J. R. et al. Reading the tea leaves: anticarcinogenic properties of (-)-epigallocatechin-3gallate. Mayo Clin Proc, 82 (6), 725-732 (2007).

3. Nanri, H. et al. Consumption of green tea but not coffee is associated with the oral health-related quality of life among an older Japanese population: Kyoto-Kameoka cross-sectional study. Eur J Clin Nutr, 73 (4), 577-584 (2019). 
4. Tomata, Y. et al. Green tea consumption and the risk of incident functional disability in elderly Japanese: the Ohsaki Cohort 2006 Study. The American journal of clinical nutrition, 95 (3), 732-739 (2012).

5. Rockwood, K. Frailty and its definition: a worthy challenge. Journal of the American Geriatrics Society, 53 (6), 1069-1070 (2005).

6. Fried, L. P. et al. Frailty in older adults: evidence for a phenotype. J Gerontol A Biol Sci Med Sci, 56 (3), M146-156 (2001).

7. Soysal, P. et al. Inflammation and frailty in the elderly: A systematic review and meta-analysis. Ageing Res Rev, 31, 1-8 (2016).

8. Lang, P. O., Michel, J. P. \& Zekry, D. Frailty syndrome: a transitional state in a dynamic process. Gerontology, 55 (5), 539-549 (2009).

9. Lorenzo-Lopez, L. et al. Nutritional determinants of frailty in older adults: A systematic review. BMC geriatrics, 17 (1), 108 (2017).

10. Kojima, G. et al. Adherence to Mediterranean Diet Reduces Incident Frailty Risk: Systematic Review and Meta-Analysis. Journal of the American Geriatrics Society, 66 (4), 783-788 (2018).

11. Rabassa, M. et al. Association of habitual dietary resveratrol exposure with the development of frailty in older age: the Invecchiare in Chianti study. The American journal of clinical nutrition, 102 (6), 1534-1542 (2015).

12. Kobayashi, S., Suga, A. K. \& Sasaki, H. S. Inverse association between dietary habits with high total antioxidant capacity and prevalence of frailty among elderly Japanese women: a multicenter crosssectional study. J Nutr Health Aging, 28, 827-839 (2014).

13. Ridker, P. M. et al. Long-term effects of pravastatin on plasma concentration of C-reactive protein. The Cholesterol and Recurrent Events (CARE). Investigators. Circulation, 100 (3), 230-235 (1999).

14. Yamada, Y. et al. Prevalence of Frailty Assessed by Fried and Kihon Checklist Indexes in a Prospective Cohort Study: Design and Demographics of the Kyoto-Kameoka Longitudinal Study. Journal of the American Medical Directors Association, 18 (8), 733 e737-733 e715. (2017).

15. Tokudome, Y. et al. Relative validity of a short food frequency questionnaire for assessing nutrient intake versus three-day weighed diet records in middle-aged Japanese. Journal of epidemiology / Japan Epidemiological Association, 15 (4), 135-145 (2005).

16. Tokudome, S. et al. Development of a data-based short food frequency questionnaire for assessing nutrient intake by middle-aged Japanese. Asian Pacific journal of cancer prevention: APJCP, 5 (1), 40-43 (2004).

17. Science and Technology agency of Japan: Standard Tables of Food Composition in Japan. 5th revised ed. Tokyo, JPB, Minitry of Finance, 2001 (Japanse).

18. Satake, S. et al. Validity of the Kihon Checklist for assessing frailty status. Geriatrics \& gerontology international, 16 (6), 709-715 (2016). 
19. Sewo Sampaio, P. Y. et al. Systematic review of the Kihon Checklist: Is it a reliable assessment of frailty? Geriatrics \& gerontology international, 16 (8), 893-902 (2016).

20. Arai, H. \& Satake, S. English translation of the Kihon Checklist. Geriatrics \& gerontology international, 15 (4), 518-519 (2015).

21. Ambagtsheer, R. C. et al. Commonly Used Screening Instruments to Identify Frailty Among Community-Dwelling Older People in a General Practice (Primary Care) Setting: A Study of Diagnostic Test Accuracy. J Gerontol A Biol Sci Med Sci, 75 (6), 1134-1142 (2020).

22. Bartali, B. et al. Low nutrient intake is an essential component of frailty in older persons. $J$ Gerontol $A$ Biol Sci Med Sci, 61 (6), 589-593 (2006).

23. Argiles, J. M. et al. Skeletal Muscle Regulates Metabolism via Interorgan Crosstalk: Roles in Health and Disease. Journal of the American Medical Directors Association, 17 (9), 789-796 (2016).

24. Alway, S. E. et al. Green tea extract attenuates muscle loss and improves muscle function during disuse, but fails to improve muscle recovery following unloading in aged rats. J Appl Physiol(1985) 2015;118(3):319-330.

25. Frei, B. \& Higdon, J. V. Antioxidant activity of tea polyphenols in vivo: evidence from animal studies. $J$ Nutr, 133 (10), 3275-3284 (2003).

26. Lagha, A. B. et al. Green tea polyphenols enhance gingival keratinocyte integrity and protect against invasion by Porphyromonas gingivalis.Pathog Dis2018;76(4).

27. Ritchie, C. S. et al. Oral health problems and significant weight loss among community-dwelling older adults. J Gerontol A Biol Sci Med Sci, 55 (7), M366-371 (2000).

28. Nanri, H. et al. Cross-Sectional Study on the Association between Dietary Non-Enzymatic Antioxidant Capacity and Serum Liver Enzymes: The Furukawa Nutrition and Health Study.Nutrients2020;12(7).

29. Liu, X., Han, D. X. \& Gao, G. W. Association between tea consumption and risk of cognitive disorders: A dose-response meta-analysis of observational studies (Oncotarget, 2017).

30. Bollwein, J. et al. Dietary quality is related to frailty in community-dwelling older adults. $J$ Gerontol $A$ Biol Sci Med Sci, 68 (4), 483-489 (2013).

\section{Figures}




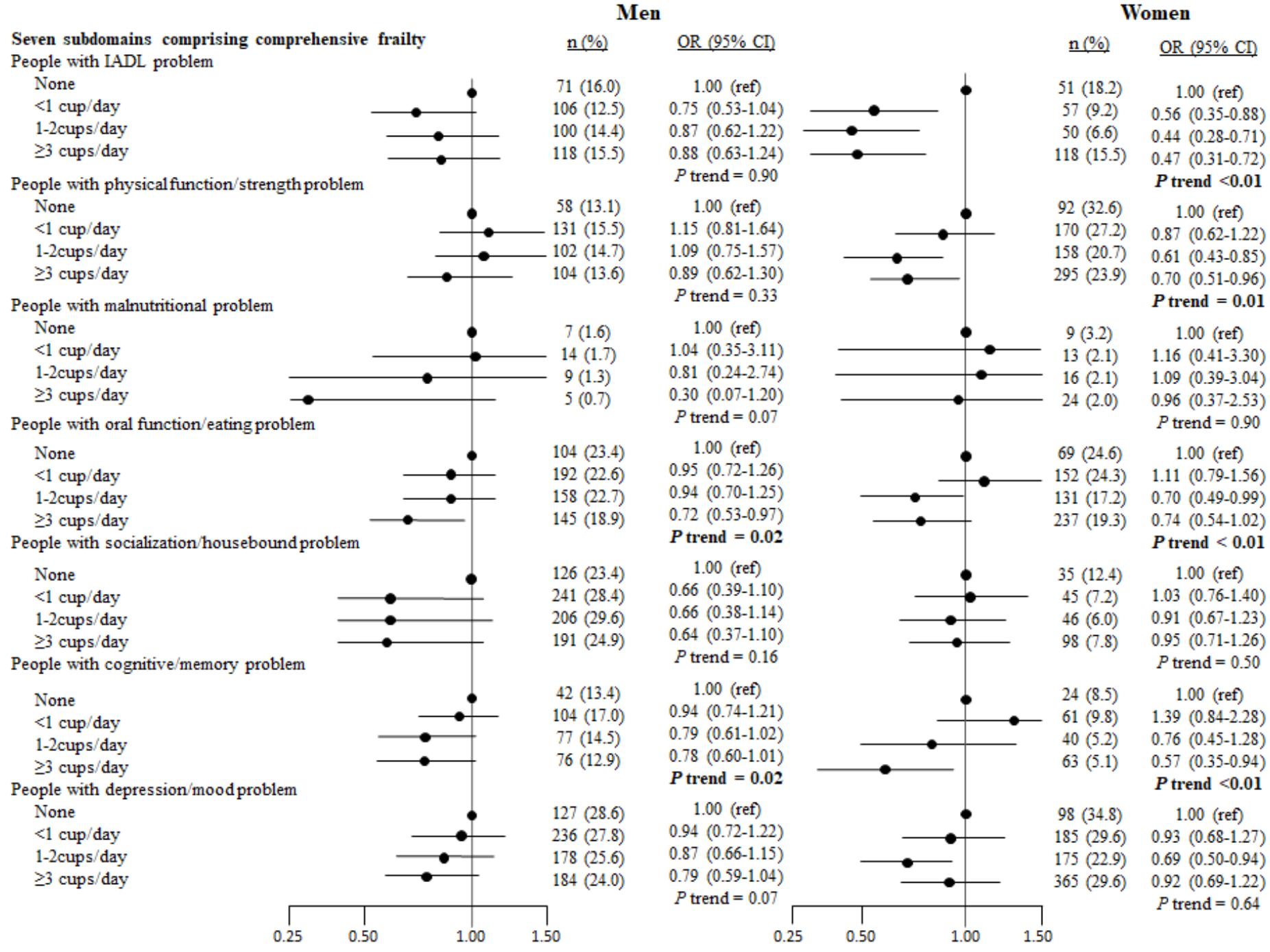

Figure 1

Multivariated-adjusted odds ratios (OR) and 95\% confidence intervals $(\mathrm{Cl})$ of the seven subdomains that constitute comprehensive frailty according to green tea consumption. Adjusted for age (years), body mass index $(\mathrm{kg} / \mathrm{m} 2)$, total energy intake ( $\mathrm{kcal} /$ day), alcohol status (everyday, sometimes, seldom, or never), smoking status (everyday, sometimes, former, or never), vegetables intake, fruits intake, and coffee consumption (almost none, $<1$ cup/day, $1-2$ cups/day, $\geq 3$ cups/day). 